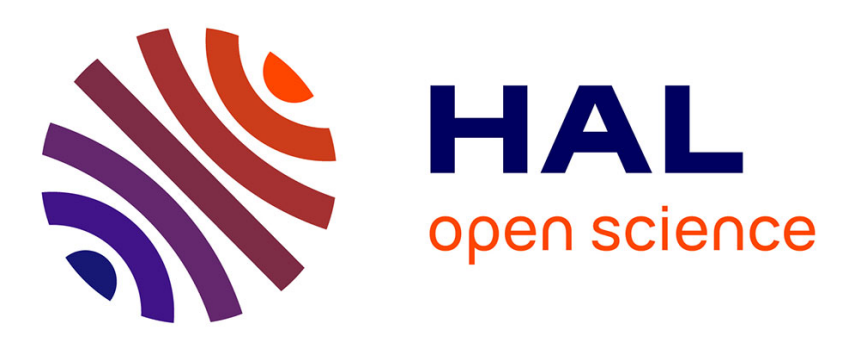

\title{
SAVING: socially aware vehicular information-centric networking
} Junaid Ahmed Khan, Yacine Ghamri-Doudane

\section{To cite this version:}

Junaid Ahmed Khan, Yacine Ghamri-Doudane. SAVING: socially aware vehicular information-centric networking. IEEE Communications Magazine, 2016, 54 (8), pp.100-107. 10.1109/MCOM.2016.7537183 . hal-01353842

\section{HAL Id: hal-01353842 \\ https://hal.science/hal-01353842}

Submitted on 15 Aug 2016

HAL is a multi-disciplinary open access archive for the deposit and dissemination of scientific research documents, whether they are published or not. The documents may come from teaching and research institutions in France or abroad, or from public or private research centers.
L'archive ouverte pluridisciplinaire HAL, est destinée au dépôt et à la diffusion de documents scientifiques de niveau recherche, publiés ou non, émanant des établissements d'enseignement et de recherche français ou étrangers, des laboratoires publics ou privés. 


\title{
SAVING: Socially Aware Vehicular Information-centric Networking
}

\author{
Junaid Ahmed Khan*† and Yacine Ghamri-Doudane ${ }^{\dagger}$ \\ *University Paris-Est, LIGM Lab, Marne-la-Vallée, France \\ ${ }^{\dagger}$ L3i Lab, University of La Rochelle, France \\ junaid-ahmed.khan@univ-mlv.fr, yacine-ghamri@univ-lr.fr
}

\begin{abstract}
Mobile devices today are constantly generating and consuming a tremendous amount of content on the internet. Caching of such "massive" data is beyond the capacity of existing cellular networks both in terms of cost and bandwidth due to its connection-centric nature. The increasing demand for content poses fundamental questions like, where, what, how to cache and how to retrieve cached content? Leveraging the shift towards content-centric networking paradigm, we propose to cache content close to the mobile user to avoid wasting resources and decrease access delays. Therefore, we present SAVING, a Socially Aware Vehicular Information-centric Networking system for content storage and sharing over vehicles due to their Computing, Caching, and Communication (3Cs) capabilities. The encapsulated $3 \mathrm{Cs}$ are exploited first to identify the potential candidates, socially important to cache in the fleet of vehicles. To achieve this, we propose a novel vehicle ranking system allowing a smart vehicle to autonomously "Compute" its eligibility to address the question, where to cache? The identified vehicles then collaborate to efficiently "Cache" content between them based on the content popularity and availability to decide what and how to cache? Finally, to facilitate efficient content distribution, we present a socially-aware content distribution protocol allowing vehicles to "Communicate" to address the question, how to retrieve cached content? Implementation results for SAVING on 2986 vehicles with realistic mobility traces suggests it as an efficient and scalable computing, caching and communication system.
\end{abstract}

Keywords-Information-Centric Networking, Vehicular Networks, Social-aware Content Distribution, Content Caching, Data Offloading

\section{INTRODUCTION}

The recent advances in the communication technologies along the soaring number of smart mobile devices results in growth of content demand by lots of consumers in closer urban proximity each with multiple portable devices. For example, large number of users on the move in an urban environment such as passengers in buses, taxis, and vehicles are interested to watch the video of a latest episode of a hot TV show/drama or a sports highlights. Provisioning of such popular content to each user require lots of redundant connections between users and the service provider, given that the content is requested by lots of spatio-temporally co-located users with similar social interests. It is now challenging for the current "connectioncentric" network infrastructure to facilitate content availability for such large number of mobile users in close proximity in an urban environment while offering attractive tariff plans supporting unlimited bandwidth. We advocate to use the recently proposed Information-Centric Networking (ICN) [1] in [2] and [3] paradigm which cater the issue by decoupling the content provider-consumer and support in-network caching at intermediate nodes.

Content caching at intermediate nodes is studied for while using different caching policies based on typical content replacement strategies such as first-in first-out (FIFO), Least Recently Used (LRU) and Least Frequently Used (LFU). This article highlights to the research community an advanced dimension of the underlying content caching challenge by posing the following fundamental questions. First, there is a need to identify the eligible candidate to cache content by finding answer to the question Where to cache?, thus finding the criteria for a node to be an important information hub in the network. Once such nodes are identified, another question follows: What and how to cache? regarding decisions based on content popularity and availability in the network. There is also a need to decide among them which nodes should keep which content to avoid redundant caching as well as different cache replacement policies. Once the content is cached in the network, the question of How to retrieve cached content? also needs to be addressed.

To address the aforementioned questions, We present a Socially Aware Vehicular Information-centric Networking model (SAVING) encapsulating them into three classes; Computing, Caching and Communications (3Cs), where mobile nodes such as vehicles with their intrinsic processing, storage and communicating capability can "Compute" their eligibility to "Cache" and "Communicate" with each other to facilitate efficient content delivery in a content-centric mobile network. We define a new notion of the computing class where a mobile node compute its eligibility to be selected as an important information hub in order to cache content. Similarly, the caching class incorporates all the questions regarding the content popularity and availability in the network including different cooperative caching schemes once the nodes compute their social importance in the network. The communication class involves different content distribution protocols where nodes communicate to retrieve the cached content from the important information hubs in the network.

SAVING presents a new concept of finding important vehicles as a ranking system comprising three novel centrality schemes, InfoRank [4], CarRank [5] and GRank [6]. Each vehicle first classify different cached information using InfoRank based on its popularity, availability and timeliness with respect to the user interest. The vehicle then autonomously computes its relative importance in the network using CarRank and GRank. CarRank allows a smart vehicle to rank itself based on its popularity with respect to the user interests, its spatio- 


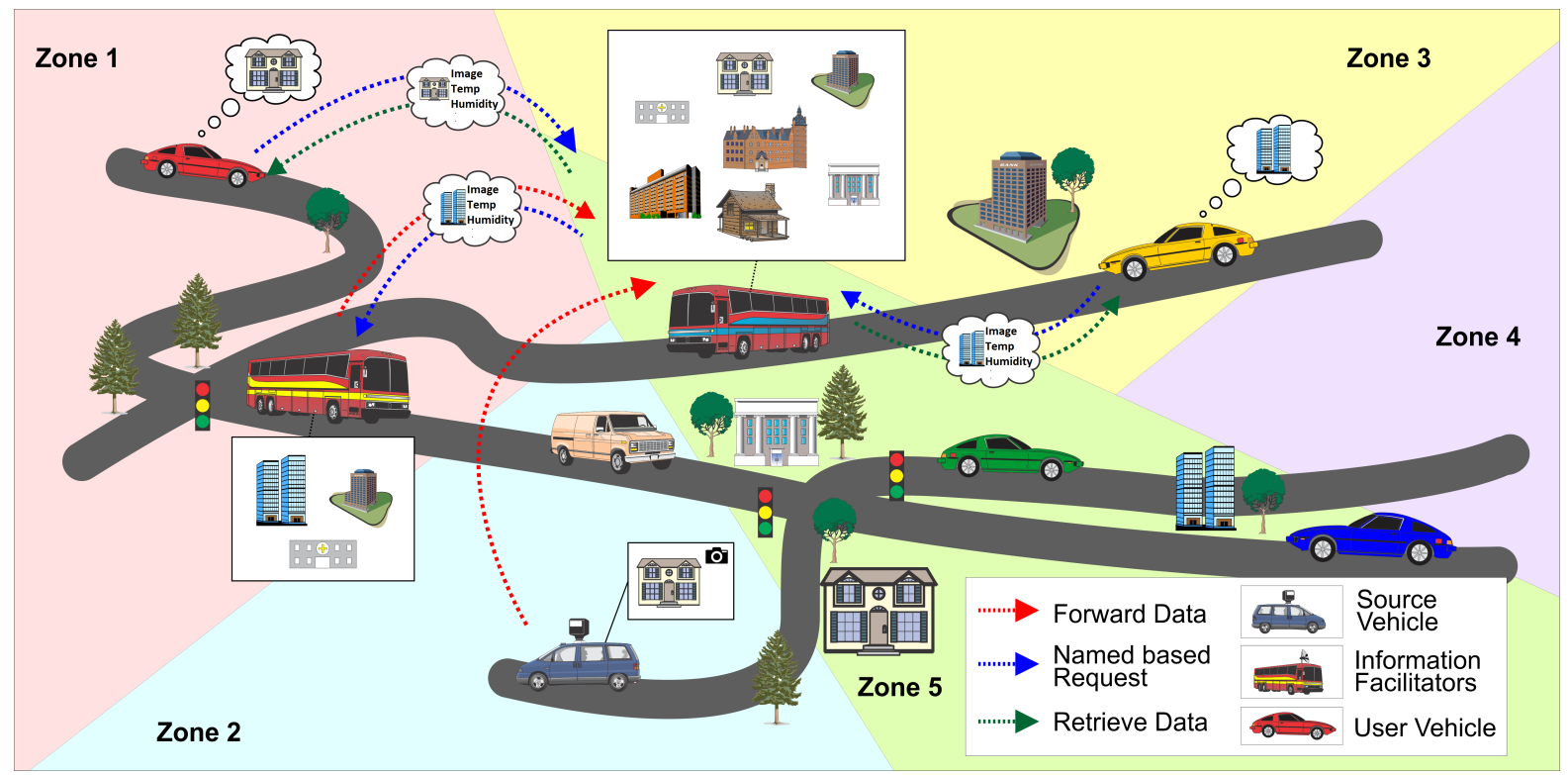

Figure 1: SAVING System Overview

temporal availability and its neighborhood connectivity as local vehicle eligibility metric. GRank considers the information reachability in an urban environment beyond local importance by allowing a vehicle to consider itself as a global "citywide"information hub to cache content in the network. Finally, we propose a social content distribution protocol where the novel vehicle centrality schemes are deployed to relay and retrieve cached content in the network.

The remaining of the article is organized as follows, the next section provides an overview of SAVING following by the description of Computing class in Section III discussing the ranking system to identify important information hubs as new trend of autonomous computing by smart vehicles. Section IV describes the Caching class explaining different criteria for to classify content. In Section V, we discuss Communication class describing the social content protocol as an example. The Section VI discuss the Performance evaluation describing the results from each class. Section VII concludes the article along some future insights.

\section{SAVING SySTEM OVERVIEW}

SAVING aims to provide a novel concept of distributed content caching and distribution framework to complement infrastructure network for urban mobile users in order to maximize content availability with minimum delays. The nameddata networking concept introduced by the information-centric networking paradigm is capable to co-exist with the mobility and intermittent connectivity challenge in mobile networks. ICN inherent in-network caching and provider-consumer decoupling maximize content availability by allowing users to retrieve content cached at "any" near-by source independent of the underlying network connectivity. We propose below an ICN enabled SAVING system by describing a use case for the location-aware content caching and distribution in an urban environment.

\section{A. Use Case: Location-aware Information Sharing}

We consider the case of location-aware content where interests for information regarding available parking lots, traffic/weather conditions, fuel prices, virtual tours to local attractions or snapshots/videos of nearby resort areas are generated by applications targeting vehicles in a given area, regardless of their IP address. To address this, SAVNG comprises a publishsubscribe ICN model allowing a mobile node such as a vehicle in our case to subscribe for the following three roles:

1) Information Provider: An information provider vehicle acts as the content source to publish content. For example, it can subscribe itself to publish sensory information collected from urban streets using the vehicle embedded cameras and sensors.

2) Information Facilitator: Vehicle responsible to collect, cache and relay data generated by information provider vehicles as well as forwards the user interest for content to "facilitate" efficient content caching and distribution.

3) Information Consumer: The vehicle subscribed to request different content from the information facilitators/providers with in the vehicular network are considered as information consumers to "pull" content in an informationcentric vehicular network.

The three distinct roles are defined since certain vehicles can be subscribed only as consumers or providers, therefore not participating to facilitate other subscribers in the network. Each ICN enabled vehicle maintains three routing parameters:

- Forwarding Information Base - FIB: It resembles a routing table which maps content name components to interfaces. Each vehicle FIB is populated by the routes discovered using our proposed centrality-based interest/data forwarding protocol.

- Pending Interest Table - PIT: It keeps track of all the incoming interests that the vehicle has forwarded but 
not satisfied yet. Each PIT entry records the content name carried in the internet, together with its incoming and outgoing interface(s).

- Content Store - CS: It is a temporary cache to store content each intermediate vehicle has received while forwarding content. Since a named-data packet is meaningful independent of where it comes from or where it is forwarded, it can be cached to satisfy future interests.

An overview of the proposed SAVING system is illustrated in Figure 1. The "User Vehicle" plays the role of a consumer vehicle interested for information regarding a location in particular zone, assuming the city is divided into different urban zones following lets say, an ICN hierarchical naming convention. It forwards the interest to an information facilitator vehicle in range which subsequently facilitate by caching and providing the desired content. The "Source vehicle" acts as the information provider by providing the content to the information facilitators responsible for the content delivery in the network.

\section{COMPUTING - Where TO CACHE?}

The identification and selection of suitable vehicles to cache content among the fleet of thousands vehicles poses an economic and bandwidth challenge along the inherent issue of mobility and intermittent connectivity. The challenge exists in finding the right set of vehicles available at the right time and place for efficient data collection, storage and distribution through low-cost inter-vehicle communications. We believe that of all the vehicles, only a set of appropriate vehicles can be considered important based on their daily commute while considering the popularity of its frequently visited neighborhoods. A vehicle can consider an information or location as popular if it observes an increase in the number and frequency of user interests for the associated content.

We define a novel concept of computing by allowing mobile nodes with sufficient processing, storage and communication capabilities to perform autonomous computing. A ranking system is presented as an example of such autonomous computing where the mobile nodes decides its user relevant importance in the network. Thus, we address the question of where to cache by identifying important mobile nodes as distributed information hubs in the network.

\section{A. Information Hubs Identification}

The self decision making of mobile nodes is leveraged to identify mobile nodes, important to declare themselves as innetwork information hubs. To do so, we propose two ranking schemes, CarRank as a local vehicle centrality and GRank, a global vehicle centrality scheme allowing vehicles to rank themselves independent of the infrastructure network. Each vehicle finds its centrality $C_{v}$ at the time instant $t_{k+1}$ from the known information in the current time-slot, where $t_{k}$ is the time instant at the beginning of the time-slot $\bar{t}_{k}$.

1) Local Information Hubs - CarRank: In the time evolving vehicular network topology, it is non-trivial to use the vehicle contact frequency and duration to decide its importance due to the rapid changes. To overcome this, we propose CarRank which simultaneously considers three novel albeit essential parameters, the information importance, the vehicle spatiotemporal availability and its network connectivity. The user's interest satisfaction for a content is also considered as a key metric for a vehicle's importance as it regularly responds to user interests. We integrate the social-awareness paradigm by allowing vehicles conform to large number of user interests for content in the network. The interests are assumed to be generated and received from the neighboring vehicles using multi-hop interest forwarding. We consider the following local parameters known to the vehicle for analytically finding its importance:

- Information importance: Information importance measures vehicle relevance to users for a particular content, i.e. The interest-response frequency is a vital factor to classify a content's importance. A vehicle associated to contents related to popular locations is considered as an important information hub in the network.

- Spatio-temporal availability: It reflects the socialbehavior based on the vehicle's habitual routes as a factor of the daily commute. Spatial availability reflects the vehicle's recursive presence in an area, while temporal availability refers to its relevance in time for a location.

- Neighborhood importance: Neighborhood importance shows vehicle topological connectivity in order to be capable to distribute information. An easily reachable and well connected vehicle in a network topology can act as an efficient facilitator.

The vehicle ranking algorithm "CarRank" is used for the identification of information facilitator vehicles to find the vehicles responsible as information hubs in the network. The vehicle first classify the information associated to it taking into consideration the relevance to the users interest. It then considers the associated information popularity to find its relative importance in the network using CarRank algorithm as its vehicle centrality:

$$
\begin{array}{r}
L C_{v}\left(t_{k+1}\right)=\theta \times L C_{v}\left(t_{k}\right)+(1-\theta) \times\left[\alpha f_{I}^{v}\left(t_{k+1}\right)\right. \\
\left.+\beta f_{T, X}^{v}\left(t_{k+1}\right)+\gamma f_{\Gamma}^{v}\left(t_{k+1}\right)\right]
\end{array}
$$

where $f_{I}^{v}, f_{T, X}^{v}$ and $f_{\Gamma}^{v}$ are the importance functions for the information, vehicle spatio-temporal availability and vehicle neighborhood respectively. Each function's contribution is normalized by the terms $\alpha, \beta$ and $\gamma$, where $\alpha+\beta+\gamma=1$, where $\theta \in[0,1]$ allows the vehicle to increase its importance with respect to the previous time-slot. The impact of each parameter differs with respect to different applications. For example, if the vehicle is located in a better connected neighborhood, it can easily spread information. Therefore, the corresponding vehicle weights the information importance along the neighborhood more than the spatio-temporal availability.

2) Global Information Hubs - GRank: Inspired from the concept of communicability in complex networks [7], GRank, a global vehicle centrality scheme allows a vehicle to use a new stable metric named "Information communicability" to rank different locations in the city and rank itself accordingly. Using GRank, the vehicle finds each location reachability and 
popularity taking into consideration the user interest satisfaction related to the location. It also considers its mobility pattern between different locations in the city along its availability in each location. Vehicles available in popular locations in the city qualify as important information facilitator vehicles with higher vehicle centrality score in the network. We can identify popular locations in the city with the maximum global centrality with respect to all information facilitators. However, popularity of locations depends on several factors such as the information-type depending on the application requirements as well as time of the day. Similarly, we can use the maximum location importance to identify popular neighborhoods for a longer time span.

The vehicle centrality function at the time instant is given as the average information global centrality for all associated locations. For a vehicle, the vehicle global centrality $G C_{v}\left(t_{k+1}\right)$ for the next time instant $t_{k+1}$ is updated as the Exponential Weighted Moving Average (EWMA) function of the current and previous global centrality as shown in the relation below:

$$
G C_{v}\left(t_{k+1}\right)=\theta \times G C_{v}\left(t_{k}\right)+(1-\theta) \times f_{G}^{v}\left(t_{k+1}\right),
$$

where $\theta \in[0,1]$ is the tuning parameter which allows the vehicle to adjust its importance with respect to the previous time-slot, $G C_{v}\left(t_{k}\right)$ is the vehicle global centrality at the beginning of the current time-slot and $f_{G}^{v}\left(t_{k+1}\right)$ is the vehicle global centrality computed at the end of the current time-slot.

The difference between both CarRank and GRank can be explained by the fact that each interest specifies two satisfaction deadlines $I_{\max }$ and $I_{\min }$, where $I_{\max } \geq I_{\min }$ indicates the maximum and minimum threshold time to provide the corresponding content. Thus in case the interest cannot be satisfied by a local facilitator vehicle (CarRank based) in the vicinity by an initial threshold $I_{m i n}$, the interests can be forwarded to more globally central vehicles (GRank based) till $I_{\max }$; the maximum interest deadline to avoid bandwidth and time utilization.

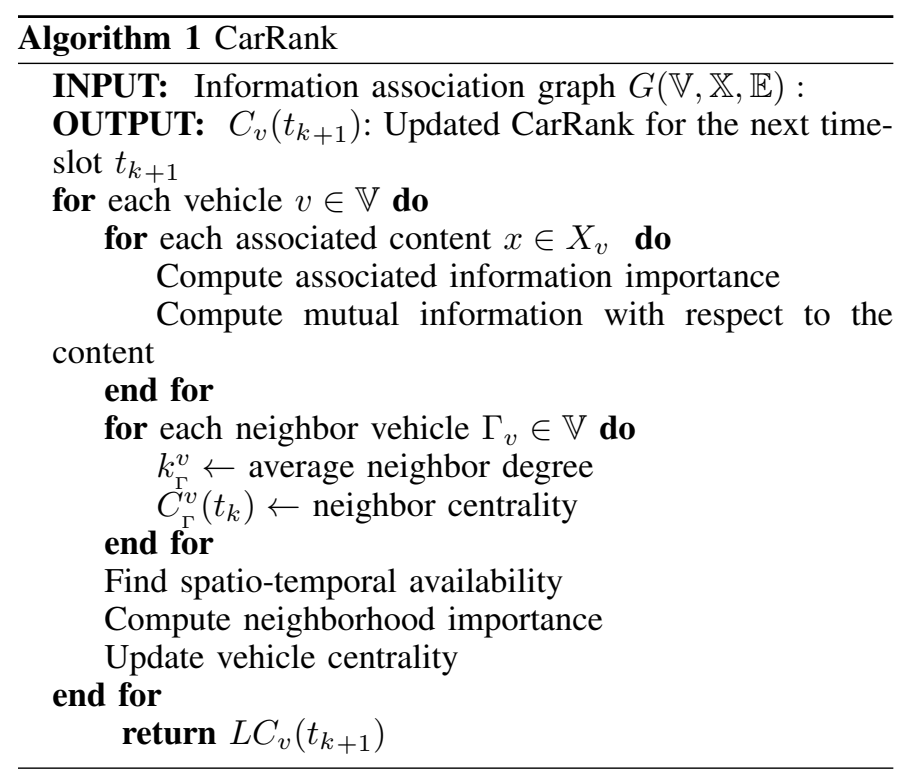

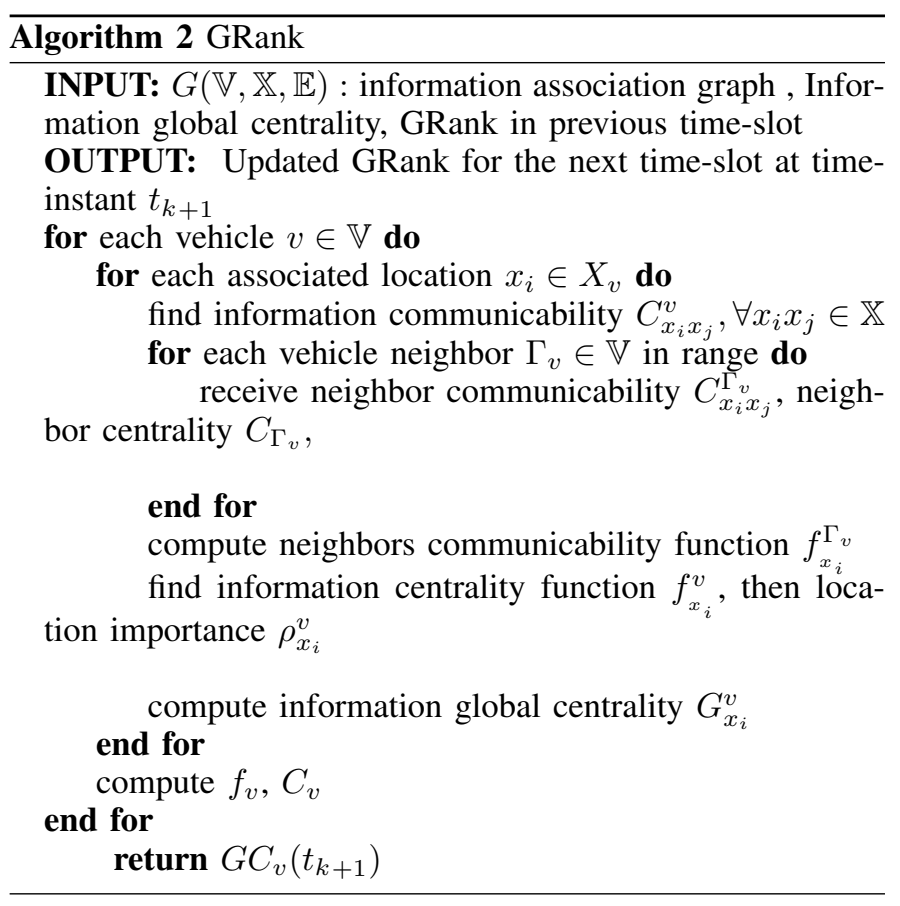

\section{CACHING - WhAT AND HOW TO CACHE?}

In this section we discuss a novel approach for content cache management by classifying the cached content importance with respect to the intended user. Lets assume vehicles encountering each other in a vehicular network constantly receives interests for content from neighboring vehicles regarding different information. Some of such information can be of more importance to the intended users in the network which the vehicle can easily recognize from the amount of user interests received for it. Therefore, a vehicle can consider an information popular if it observes an increase in the number of user interests for the associated content. We assume that it is capable of recording the time and position each time it responds with the desired content to a user interest. Thus, SAVING incorporates a novel distributed algorithm InfoRank with the concept of enabling a mobile node to rank important information associated to it based on the satisfied user interests and the information validity scope.

\section{A. Interest Satisfaction Frequency}

We define interest satisfaction frequency as the frequency of user interests satisfied in the previous time-slot as the ratio of the number of successful responds in the previous time-slot and the total successful responds for the content associated to the vehicle. Thus, the vehicle regularly updates each content importance value depending on the interest satisfaction frequency. We assume that each vehicle is capable to record the time and position each time it responds as the content provider to a user interest. Interest for each content specify the temporal scope of information validity, For instance, road congestion information is only valid during congestion. Therefore, it should be ensured that the information importance is not substantially augmented after the desired deadline. 


\section{B. Information Timeliness}

The information timeliness $\tau$ is the measure of the temporal information validity scope which can be adjusted by a tuning parameter depending on the application needs (E.g. 1 hour for accident information validity). If there are no active interests and the average interest validity time has passed, the information importance adapts an exponential delay since the information is of less importance in the network. However, $\tau$ is set to unity for content to be always available in the network.

The content importance depends on its importance at the beginning of the time-slot. If it is not responded in the previous slot, then the content importance is not increased unnecessarily. We also consider the percentage of time the vehicle itself acted as the original source for any content. InfoRank is updated regularly to ensure the content relevant to vehicle retain its value in case the vehicle does not respond in the previous slot. The interest later in time could finally route to the vehicle which maintains its value as the original source for particular content. A tuning parameter decides the importance value with respect to the associated content in cache. For all contents associated to a vehicle, we also consider the ratio of missed interest to the total interests received by the vehicle. Missed interest provides the vehicle reliability regarding successful respond to the incoming interests.

To summarize with an example, Assume a vehicle visiting an area at some future time-slot place an interest for the content regarding that area. This interest is propagated to potential facilitator vehicle. Each vehicle upon receiving the interest message checks its cache to find a match regarding the desired content. In case the interest could not be satisfied, it is forwarded to neighboring vehicles. In case a match is found, it responds to the interest message by providing the corresponding content where each vehicle compute its cached information importance by finding its respective InfoRank score. Once the information importance is agreed between different facilitators, collaborative caching between nodes (i.e. P2P networking) can be ensured by mutually respecting a social norm to avoid redundant content caching in the network.

\section{Communication - How to Retrieve?}

In this section we discuss the efficient retrieval of the cached content in the network by focusing on the communication aspect. For this reason, we present an idea of social aware content caching and distribution scheme where the consumer "pulls" content of interest cached at important information facilitator vehicles in the network. We use the above mentioned two novel vehicle centrality schemes to identify important information facilitator vehicles based on cache management for content suggested by InfoRank scheme.

\section{A. Content Distribution Protocol}

The centrality-based content distribution protocol leverage the facilitator centrality to forward consumer interests for content as well as route the content from the corresponding information providers. The provider as well as the consumer search for a near-by information facilitator vehicle using its centrality score to forward interest/content. We propose a hybrid content distribution protocol with an ICN inherent pull

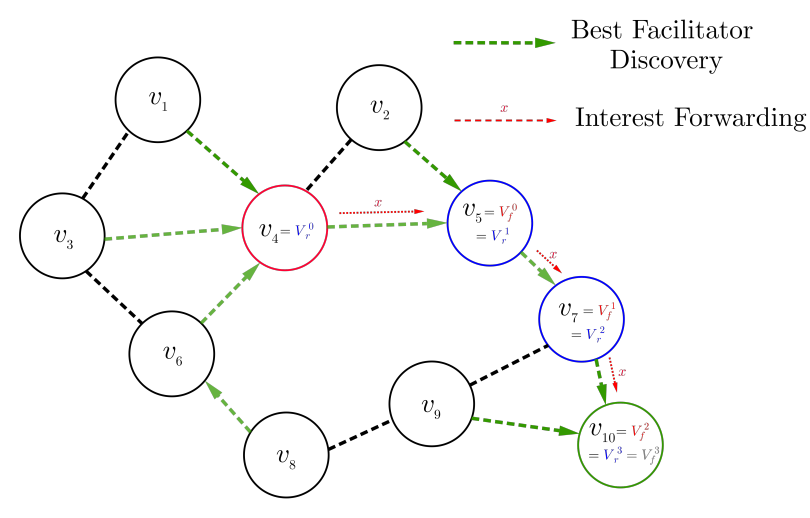

Figure 2: Facilitator Discovery Process

based content retrieval for the consumer and a push based approach for the provider to publish content.

Information Facilitator Discovery: The facilitator discovery process allows a vehicle to search in its vicinity the highest centrality facilitator vehicle using the FACILITATOR() function. It compares the facilitator centrality score of all the neighboring vehicles and returns the best facilitator centrality vehicle among the vehicles in range for a vehicle. The PROVIDER() function assigns a vehicle to be the provider vehicle to publish the content for the consumer vehicle. The publishing of content by the provider can be either solicited or non solicited. In the case of solicited interest, the provider can publish content destined for the vehicle with a near-by information facilitator using PUBLISH() function. Similarly, a nonsolicited publish with a near-by facilitator can be performed by an information facilitator discovery process initiated anytime by the information provider. The CONTENT() function is used for the content availability check at each intermediate vehicle Content Store (CS).

Figure 2 depicts the social content distribution protocol. Consumer vehicle $v$ generates interests for content as INTEREST () towards the best ranked facilitator in the vicinity. The facilitator discovery process continues to search for the content at each intermediate relay vehicle by constantly discovering the next best ranked vehicle in the vicinity of each intermediate relay vehicle. Thus, each relay vehicle becomes the responsible vehicle to facilitate the content. If it is unable to find the content in its CS, it performs a facilitator discovery to find a vehicle with higher facilitator centrality score and a Pending Interest Table (PIT) entry is created. The process is repeated at each intermediate facilitator till either the desired content is found or there are no more facilitators to discover.

The convergence of the facilitator discovery process is twofold, the first obvious convergence occurs when the desired content is available at the corresponding facilitator. In this case, the content is published at the consumer vehicle following a reverse path to the initial requester using breadcrumbs left in the PIT at each intermediate node. The intermediate vehicles subsequently populates the corresponding Forwarding Information Base (FIB) entry for the content. In case the content is not available and there are no further facilitators to discover, the responsible vehicle declares itself as the content 
provider to publish content at the consumer vehicle.

\section{PERFORMANCE EVAluAtion}

The performance of the SAVING is validated by a set of simulations under a realistic mobility scenario using traces from Cologne, Germany as an accurate mobility trace available for Vehicular Environment [8]. The number of vehicles in each region vary at different time of the day. We analyze up to 2986 vehicles in the entire simulation duration with one second of time granularity. The Cologne city center is simulated for one hour by clustering the $6 x 6 \mathrm{~km}^{2}$ The number of regions can vary between different cities depending on the size, though we divide Cologne into 36 neighborhoods. The urban roads with vehicle communication range around $300 \mathrm{~m}$ is considered. The Nakagami path loss model in combination with Log-distance propagation model to cater for the impact of buildings and other obstacles.

\section{A. Simulation Scenario}

We simulate a urban vehicular network using the ndnSIM (http://named-data.net/techreports.html) to integrate the Named Data Networking (NDN) communication model. The simulation scenario implements the following applications:

1) Consumer: Consumer vehicles are the potential users planning to visit an area. Each consumer vehicle generates an interest for a content associated to a location in the city, which is routed to provider vehicles.

2) Provider: We define a vehicle to be the content provider in the network for the areas visited in a time-slot before the consumer interest generation time. The areas visited are considered as locations associated with the provider.

3) Facilitator: Vehicles satisfied incoming requests generated form consumers regularly computes their centrality score to consider themselves as information facilitators. Similarly, constant content forwarding and cache hits also counts towards the facilitator centrality score.

We associate each vehicle with a set of different locationdependent content as its cached content. Each vehicle is enabled to randomly generate interest with varying frequency at different time intervals for different (predefined) content as consumer. The interest profile characteristics is two-fold. First we evaluate an information using InfoRank considering its popularity based on the number, frequency and spatiotemporal validity of generated interests for the content. Then, considering the cached content importance, we imply our ranking scheme CarRank and GRank to evaluate the interest profile for the associated vehicle.

We assume the interests follow a Zipf distribution, where we observe frequent interests for content regarding popular locations.

\section{B. Simulation Results}

For better performance analysis of the proposed SAVING system in different simulation scenarios, we compare it with the state of the art social-aware routing schemes. Such schemes typically rely on centrality schemes such as Degree, Closeness,

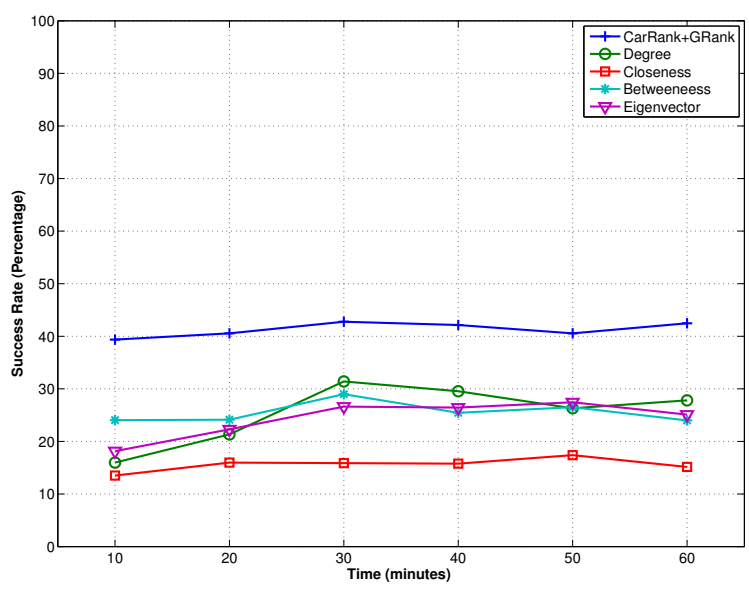

Figure 3: Success rate conparison for the consumer interests satisfied over time using different centrality schemes for content distribution

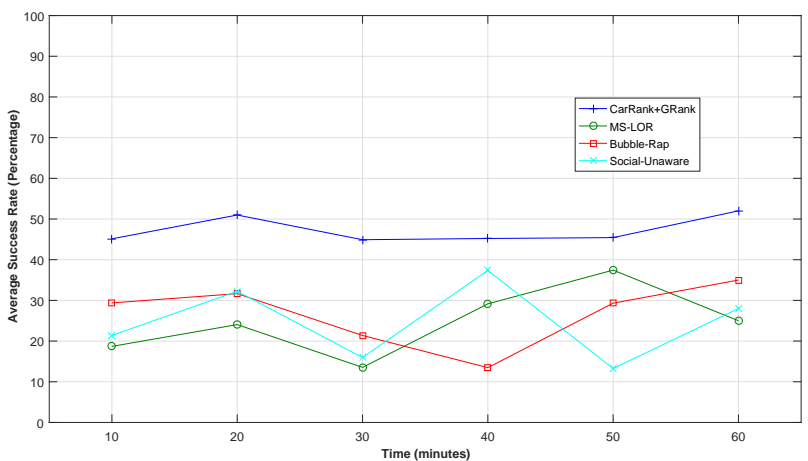

Figure 4: Success rate comparison of SAVING for the consumer interests satisfied over time withs social-aware DTN schemes and a social-unaware variant for content distribution

Betweenness and Eigenvector centrality. Therefore, we perform a comparative analysis of the proposed vehicle centrality based routing with the benchmark centrality schemes with the following performance metrics:

- Success rate for satisfying consumer interests in the network

- Aggregated content store (cache) hit rate at the information facilitators

1) Success Rate: Success rate refers to the percentage of the generated consumer interests successfully satisfied over the entire simulation duration.

(a) Benchmark Centrality Schemes Comparison: The proposed vehicle centrality based content distribution is compared with the state of the art centrality schemes as benchmark. Figure 3 shows the percentage of consumer interests for different locations successfully satisfied by the corresponding information facilitators/providers. We observe that forwarding the interest towards a socially important vehicle using CarRank 


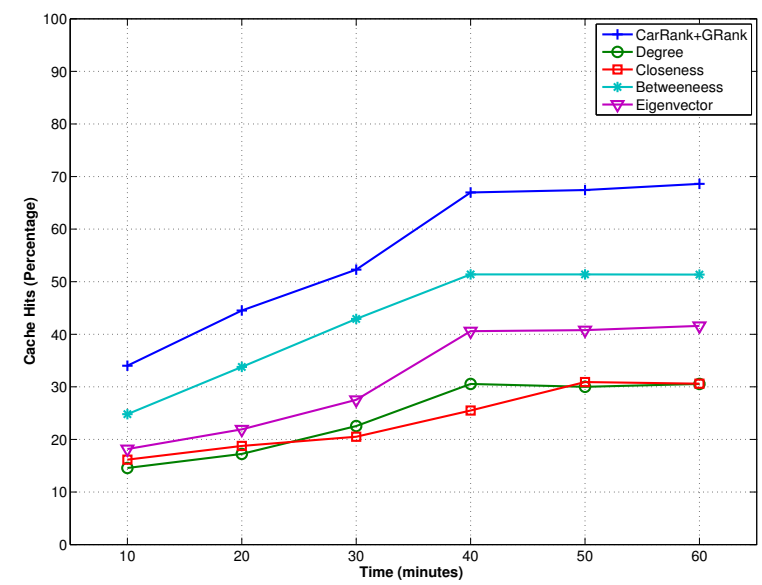

Figure 5: Comparison result of the percentage of the cache hits over time at the information facilitators selected using different centrality schemes for content distribution

and GRank as a metric results in more number of successful interest satisfaction. The vehicles identified by the proposed vehicle centrality metric satisfied around $40 \%$ of interests compared to other centrality metrics despite high mobility and intermittent connectivity. It is because typical centrality schemes only takes into account physical topology towards computing a node importance in the network, ignoring the satisfied user interests.

(b) Socially aware DTNs and Social Unaware Schemes Comparison: We also compare the success rate of SAVING with two relevant socially-aware DTN routing schemes MSLOR [9] and Bubble-Rap [10] as well a variant without considering social awareness. BubbleRap uses a hybrid metric based on community and betweenness centrality where MSLOR uses a three-layer social metric based on degree centrality. The social-unaware approach implements interest flooding in which each consumer re-broadcasts interest to all of its neighbors except the one from which it received. Figure 4 depict the results from the comparative analysis. We observe that using CarRank and GRank based routing yield a success rate around $40 \%-50 \%$ where social-aware DTN schemes achieve a maximum of $38 \%$ at 50 minutes from ML-SOR. An interesting observation made is the stability of SAVING unlike other schemes. It is because thy fundamentally rely on centrality measures which assume a static graph topology with respect to time. Moreover, the host-centric nature instead of content-centric approach with no in-network caching support limits DTN capability to maximize content availability. Thus, a content distribution based on adapted metrics (such as CarRank and GRank) better cope with the dynamic nature of vehicular network.

2) Cache-hits: We evaluate the ICN built-in feature of in-Network caching at intermediate nodes at the selected facilitator vehicles. For this purpose, we compute the cache hit rate at the facilitator vehicles. A second successful response by a vehicle for the same content is considered a cache hit. The cumulative cache hit rate is computed for the entire simulation duration. Figure 5 shows the cache hit rate for the facilitator vehicles identified by each centrality scheme. The vehicles identified by our proposed vehicle centrality scheme yield a higher hit rate than all the other schemes. This is because we consider content popularity as a key factor, thus, the vehicle containing important information responds and subsequently cache more frequently compared to other vehicles.

We also observe that the vehicles identified using betweeness centrality follows our proposed scheme yielding better cache hit rate due their frequent availability as intermediate bridges at most of the shortest paths, thus allowing them to cache more content. Moreover, the intermediate facilitators identified by our vehicle centrality scheme cached more important content due to their better neighborhood connectivity and spatio-temporal availability in the network. This proves that innetwork caching offered by ICN along the proposed vehicle centrality scheme overcomes the mobility and intermittent connectivity constraints in vehicular network for efficient content distribution.

\section{CONCLUSIONS AND OPEN RESEARCH ISSUES}

We proposed SAVING as an alternate solution to leverage smart vehicles with their caching, computing and communicating capabilities to facilitate content availability for an urban mobile user with minimum content access delay. SAVING is a socially-aware Vehicular Information-centric system focusing the research community interest towards the application of combining socially aware content distribution scheme with the information-centric networking paradigm. We explored possible answers to the fundamental questions of where, what and how to cache content in mobile networks under an increasing growth of mobile traffic. Moreover this article highlighted another perspective by equally considering the efficient content retrieval by caching at vehicles. The suggestion for vehicles can also be generalized for all sort of mobile nodes depending on the node computing, caching and communication (3Cs) capabilities.

Open research issues include efficient social aware routing strategies, flexible and scalable naming scheme for novel applications and the possibilities to support high bandwidth consuming content video streaming in content-centric networking paradigm. However each of the 3Cs still lacks exploration by the current research requiring intelligent algorithms for nodes to make real-time decisions regarding the cached content. Similarly the need for distributed cache management schemes with collaborative content replacement strategies with redundancy avoidance for the daily massive content generated needs to be thought about. Thus, we invite the research community to explore the new trends exploiting socially-aware network computing, caching and communication in a contentcentric approach to overcome the limitations of the existing connection-centric approach.

\section{REFERENCES}

[1] Ahlgren, Bengt, Christian Dannewitz, Claudio Imbrenda, Dirk Kutscher, and Börje Ohlman. "A survey of information-centric networking." Communications Magazine, IEEE 50, no. 7 (2012): 26-36.

[2] Amadeo, Marica, Claudia Campolo, Antonella Molinaro, and Giuseppe Ruggeri. "Content-centric wireless networking: a survey." Computer Networks 72 (2014): 1-13. 
[3] Gerla, Mario, Eun-Kyu Lee, Giovanni Pau, and Uichin Lee. "Internet of vehicles: From intelligent grid to autonomous cars and vehicular clouds." In Internet of Things (WF-IoT), 2014 IEEE World Forum on, pp. 241-246. IEEE, 2014.

[4] Khan, Junaid Ahmed, Yacine Ghamri-Doudane, and Dmitri Botvich. "InfoRank: Information-Centric Autonomous Identification of Popular Smart Vehicles.” In Vehicular Technology Conference (VTC Fall), 2015 IEEE 82nd, pp. 1-6. IEEE, 2015.

[5] Khan, Junaid Ahmed, and Yacine Ghamri-Doudane. "Car Rank: An Information-Centric Identification of Important Smart Vehicles for Urban Sensing." In Network Computing and Applications (NCA), 2015 IEEE 14th International Symposium on, pp. 184-191. IEEE, 2015.

[6] Khan, Junaid Ahmed, Yacine Ghamri-Doudane, and Dmitri Botvich. "GRank-An Information-Centric Autonomous and Distributed Ranking of Popular Smart Vehicles." In 2015 IEEE Global Communications Conference (GLOBECOM), pp. 1-7. IEEE, 2015.

[7] Estrada, Ernesto, and Naomichi Hatano. "Communicability in complex networks." Physical Review E 77, no. 3 (2008): 036111.

[8] Uppoor, Sandesh, Oscar Trullols-Cruces, Marco Fiore, and Jose M Barcelo-Ordinas. "Generation and analysis of a large-scale urban vehicular mobility dataset." Mobile Computing, IEEE Transactions on 13, no. 5 (2014): 1061-1075.

[9] Socievole, Annalisa, Eiko Yoneki, Floriano De Rango, and Jon Crowcroft. "ML-SOR: Message routing using multi-layer social networks in opportunistic communications." Computer Networks 81 (2015): 201219.

[10] Wei, Kaimin, Xiao Liang, and Ke Xu. ”A survey of social-aware routing protocols in delay tolerant networks: applications, taxonomy and designrelated issues." Communications Surveys \& Tutorials, IEEE 16, no. 1 (2014): 556-578. 